\title{
The Analysis of COVID-19 Prevention Experience Using DEMATEL-Based ANP
}

\author{
Cheng-Wen Lee ${ }^{1}$ and Yi Tang $\mathrm{Hu}^{2}$
}

\begin{abstract}
This research uses the Decision Making Trial and Evaluation Laboratory and Analytic Network Process (DEMATEL-Based ANP) analysis to examine the Taiwan's experience for preventing COVID-19. We use the eight factors to fight COVID-19 as listed by Taiwan's Ministry of Health and Welfare such as SARS experience, Central Epidemic Command Center, Information Transparency, Good resource allocation, Timely border control, Smart community transmission prevention, Advanced medical technology, Good etiquette of a citizen. The result findings present the key factors for success and their interrelationships with each other. This study would be expected as a reference for suggesting other countries those are working together to fight COVID-19.
\end{abstract}

JEL classification numbers: A10, D71, H51.

Keywords: Key success factors, COVID-19, DEMATEL-based ANP.

${ }^{1}$ Professor, Department of International Business, Chung Yuan Christian University, Taiwan.

2 Ph.D program in Business, College of Business, Chung Yuan Christian University, Taiwan.

Article Info: Received: November 23, 2020. Revised: December. 11, 2020.

Published online: December 14, 2020. 


\section{Introduction}

Clusters of unexplained pneumonia had been found in Wuhan, Hubei, China, in December 2019. These initial cases of the epidemic were mostly related to the history of activities in Wuhan South China Seafood City. By January 9, 2020, Chinese officials announced that the pathogen was a new type of coronavirus. The epidemic quickly spread to other provinces and cities in China and around the world and proved to be possible for human-to-human transmission. In order to monitor and prevent this emerging infectious disease, Taiwan announced "Severe Special Infectious Pneumonia" (COVID-19) as the fifth statutory infectious disease from January 15, 2020, and the first case was diagnosed on January 21, 2020. The confirmed case of immigration from abroad and the first local case confirmed on January 28 , which is a family group infection caused by immigration from abroad. The new coronavirus pathogen's characteristics are still under study. Its family is an important pathogen that causes human and animal diseases. It is a group of single-stranded positive-stranded RNA viruses with a mantle. The appearance is round. The crown-like protrusions can be seen under an electron microscope, hence the name. Coronavirus can cause diseases of humans and vertebrates and is a zoonotic disease. Human infection with coronavirus is mainly caused by respiratory symptoms, including nasal congestion, runny nose, cough, fever, and other general upper respiratory tract infection symptoms. Some cases may have severe pneumonia and respiratory failure (https://covid19.mohw.gov.tw/).

The new crown virus is sweeping the world, and the number of confirmed cases in the country is increasing. This has also made many people feel restless. As long as they feel unwell in their throats and start to cough, they can't help but wonder if they have also contracted new crown pneumonia. Although the symptoms of new coronary pneumonia, colds, and flu are very similar, there are still some differences. Let's quickly understand what are the main symptoms of these three diseases.

Colds rarely cause systemic symptoms, mainly upper respiratory symptoms such as nasal water, nasal congestion, and cough, and are less likely to have fever, but children under three years of age may have fever. The symptoms of coronavirus and flu tend to be systemic, making you feel very tired and painful, and you can basically only sleep in bed. The above only lists the main symptoms of each disease. The indication "No" does not mean that the symptoms will not appear, because each person's symptoms may be slightly different. If you feel unwell, please consult a doctor.

There is currently no vaccine available to prevent this new type of coronavirus (SARS-CoV-2) infection, and directly contact with the secretions of suspected which is currently known as COVID-19 cases and the prevention of droplet transmission should be avoided. Although the rest of the world is still suffering from the many adverse effects of the novel coronavirus (COVID-19) pandemic, Taiwan has successfully shut out COVID-19 through its early and ongoing interventions. The Taiwan Centers for Disease Control and Prevention launched the Central Epidemic Command Center (CECC) on January 20, 2020. In the past 100 days, 
Taiwan has implemented border restrictions, home quarantine, mask stacking systems, and other preventive measures. Fight against this viral infection. Taiwan's achievements in stopping the nationwide outbreak have gained global recognition ("Lianhe Daily" April 29, 2020).

The CECC stated on June 7 that the global protected COVID-19 epidemic is raging, and Taiwan is still responding and controlling quickly and effectively, minimizing the impact of the epidemic on people's lives. In order to record this kind of epidemic prevention experience, the Ministry of Health, Welfare and Welfare specially integrated the decision-making procedures of epidemic prevention policies formulated by the central and local governments, which is called the "Taiwan Model". It explains Taiwan to the public and other countries in the form of a timeline. The factors for the success of epidemic prevention, the foundation of the health care system, and major policies have enabled all walks of life to understand Taiwan's public health capabilities, proving the slogan "Taiwan can help, and Taiwan is helping!"

Under the severe international epidemic situation, Taiwan's infection risk is relatively stable. After implementing forward-looking advanced deployment measures, the domestic epidemic prevention materials have gradually been filled. This virus knows no borders. As long as the global epidemic does not slow down, Taiwan will continue to face the threat of COVID-19. Due to the less demand in Taiwan, it is willing and able to help other international friends and cooperate with other countries to develop masks, therapeutic drugs, and epidemic prevention technologies to prevent congestion (https://www.cdc.gov.tw/).

\section{Status of Taiwan's Prevention Method}

In response to the World Health Organization (WHO) denying that Taiwan has warned that COVID-19 may spread from person to person, the Central Epidemic Command Center issued an official statement on April 11, 2020, as follows:

1. The Department of Disease Control has learned from the Internet that at least seven cases of atypical pneumonia (often refers to as SARS) have occurred in Wuhan, China. SARS is a serious human-to-human disease caused by the coronavirus.

2. Due to the SARS experience, we are highly vigilant about the news of the epidemic, so we immediately notified the World Health Organization (WHO) "International Health Regulations (IHR)" contact window by email on December 31, 2019, requesting it Provide further information. As the information on the epidemic situation was unclear at the time, various rumors were abounding, in order to make all parties highly vigilant, especially the timing of the outbreak of the epidemic was close to the Chinese New Year when a large number of people moved. In order to be cautious, we specifically mentioned "atypical pneumonia" in the email, especially "patients have been treated in isolation," so that public health professionals can determine the possibility of "person-to-person transmission" in these cases. However, there 
are no cases in my country, and it is impossible to directly indicate that the disease has been transmitted from person to person.

3. The CDC also contacted the Chinese Center for Disease Control, hoping to obtain more information on the epidemic. However, the WHO IHR contact window only replied that it had transferred my information to the relevant unit; the Chinese side only provided us with a press release.

Since people strongly suspect that there has been human-to-human transmission and cannot be clarified through existing channels, our government immediately confirmed on the same day as the notification, according to the procedures for handling the possibility of human-to-human transmission, initiated border quarantine enhancement measures, and targeted the dispatch of flight personnel from Wuhan. Boarding and quarantine operations. The CDC also dispatched experts to Wuhan in mid-January to learn about the epidemic situation, prevention measures, and patient exposure history. Based on preliminary research, we have concluded that this pneumonia will indeed spread from person to person.

Taiwan's sound public health and medical systems, coupled with the public's acceptance of protective policies affected by the 2003 SARS epidemic, were likely to help effectively implement these policies in the first 50 days of the COVID-19 outbreak. At the same time, Taiwan's response to COVID-19 may overlook other health threats, such as seasonal flu and chronic diseases. Strategic prioritization of other public health functions and resources, as well as broader government operations, were necessary. As the outbreak continues, Taiwan will need to evaluate relevant policy decisions to maintain the system.

Taiwan has learned from the experience and lessons of SARS, and some of the successful strategies adopted during the current pandemic may provide references for other governments' policies. In countries that rely heavily on national and local actions, intergovernmental and judicial coordination, and sufficient funds are needed to ensure emergency preparedness and response capabilities. Combining public health, the integrated approach of human services and healthcare systems can improve resilience and better prepare countries for future events.

The command center pointed out that the "COVID-19 Key Decision-Making Network for Taiwan Epidemic Prevention" has a Chinese and English version of the interface, which contains four major categories: "Critical Timeline for DecisionMaking," "Successful Epidemic Prevention Factors", "Basics of Taiwan's Health and Medical System" and "Major Policies" sections:

1. Key decision-making timeline: Displays the cumulative number of confirmed diagnoses in Taiwan and internationally, and compares Taiwan's various decision-making time points to show the context of Taiwan's various advanced deployment decisions.

2. Factors for successful epidemic prevention: Summarize "SARS experience", "Central Epidemic Command Center", "Open and Transparent Information", "Good Resource Allocation", "Timely Border Control”, "Smart Community Epidemic Prevention", "Advanced Medical Technology" 8 categories, such as "Quality Citizens", are described in a simple, line-up style with pictures. The 
success of epidemic prevention depends on the concerted efforts of the central and local governments to fight the virus and the high-quality cooperation of the people.

3. The foundation of Taiwan's health and medical system: It explains Taiwan's long-term efforts in the field of medical and health care in the past, establishing excellent health and medical foundation, and implementing effective and rapid epidemic prevention measures.

4. Major policies: A detailed description of the major policy content of the epidemic prevention process. Furthermore, they verify and implement "Taiwan can help, and Taiwan is helping!"

\section{Literature Review}

Cheng, Li, and Yang (2020) show how quick and efficient was the Taiwanese government during the pandemic outbreak. At the early stage of the outbreak, the Taiwanese Government focused on three strategies such as real-time surveillance with rapid risk assessment, border control and quarantine, and laboratory capacity building. Husnayain, Fuad, and $\mathrm{Su}$ (2020) propose the potential use of Google Trends (GT) data for the specific locations and subregions in Taiwan nationwide to keep an eye on public restlessness toward COVID-19 infection in Taiwan. They used search terms related to the coronavirus, handwashing, and face masks. Results showed that searches related to COVID-19 and face masks in Taiwan rapidly increased following the announcements of Taiwan's first imported case and reached a peak as locally acquired cases were reported. However, searches for handwashing gradually increased during the period of face-mask shortage.

Chin et al. (2020), studying the Taiwanese Government's response to the novel pandemic, argue that enhance Traffic Control Bundling (eTCB) could interrupt the community-hospital-community transmission cycle, thereby limiting the impact of the pandemic. eTCB's success derived from ensuring that Health Care Workers and patients were protected from fomite, contact, and droplet transmission within hospitals. Evidencing eTCB effectiveness is Taiwan's success to date in containing and controlling the community-hospital-community transmission cycle.

Ko et al. (2020) scrutinize the variety of information sources used by the healthcare workers and the general public during the COVID-19 pandemic. They find that the non-healthcare workers receiving COVID-19 from medical staff in health care settings had better psychological well-being. Research also demonstrated that COVID-19 affects older adults more as well as people with comorbidities, such as hypertension, cardiovascular disease, diabetes, chronic respiratory disease, and chronic kidney disease. These groups of people have a greater need for health information. Thus, the authors suggest that all medical staff, not only frontline staff, must have complete and accurate information on COVID-19 to educate the public; the Internet and television are practical vehicles for public health education during this pandemic.

Huang (2020) provides some background on the systems and organizations that 
helped Taiwan streamline a task force (command center) in a timely manner and reviews some of the conditions and practices that highlight the positive interplay between government initiatives and public support. The author found that Taiwan experiences a "collaborative governance" model in which government initiatives and collaboration from other sectors worked together to prevent the spread. A combination of well-implemented measures to block, track, and isolate possible sources of infection, along with high public compliance, helped Taiwan to be among the best global responses to COVID-19 pandemic.

Hsieh et. al. (2020) show that Taiwan was one of the first countries to respond to the COVID-19 pandemic. It responded by monitoring travelers with enhanced measures and procedures, including the use of thermal screening systems to check suspected cases. Moreover, the authors presented two action categories exemplified by Taiwan that seem to be unique in the beginning phase of the COVID-19 pandemic compared to the rest of the world-universal hygiene and mass masking - that could potentially influence the transmission of other infectious diseases. It appears evident that the combination of mass public masking and hygiene provided a very significant result.

Lee (2020) state that Taiwan takes credit for its effective response to coronavirus disease 2019 (COVID-19). As of May 9, the laboratory-confirmed cases were 440 in number, with low mortality rate. Nearly $80 \%$ of all cases were imported. The success of the epidemic control has resulted from the post-SARS self-alert and selfdiscipline of the residents, who voluntarily put on face masks, wash hands properly, and practice social distancing. Another contribution from the public is the wide application of big data analysis and advanced information and communication technology (ICT). The management of the pandemic crisis is widely believed to be a blueprint for many other countries.

Peng (2020) claim Taiwan's response to the COVID-19 pandemic has not only protected its citizens from the rapid community spread but has also deserved to be part of World Health Organization (WHO). The author believes that Taiwan's approach in managing the pandemic has made it a viable player on the international stage.

Lin et. al. (2020) analyze the use of the National Health Insurance database and critical policy decisions made by Taiwan's government during the first 50 days of the COVID-19 outbreak. They found that Taiwan's robust public health and healthcare systems, combined with public acceptance of protective policies influenced by the 2003 SARS outbreak, likely bolstered efficient implementation of policies in the first 50 days of the COVID-19 outbreak. At the same time, Summers et al. (2020) mention that Taiwan's response to COVID-19 might have overshadowed other health threats, such as seasonal influenza and chronic diseases. Strategic prioritization of other public health functions and resources and broader government operations will be necessary. As the outbreak continues, Taiwan will need to evaluate associated policy decisions to sustain the system.

Ching-Fu et. al (2020) analyzing Governments regulatory actions during the novel pandemic reveal that Taiwan's experience in fighting COVID-19 presents an 
alternative model to China's myth of authoritarian effectiveness. Their study also points to the failure of the current international public authority in moving beyond the political boundaries that diseases neither recognize nor respect.

\section{Methodology}

This research uses the Decision Making Trial and Evaluation Laboratory and Analytic Network Process (D-ANP) analysis. Because DEMATEL's total impact matrix is used as the basis for generating the criterion unweighted supermatrix, the production of the paired comparison questionnaire is exempted. Respondents only need to fill in the direct impact matrix.

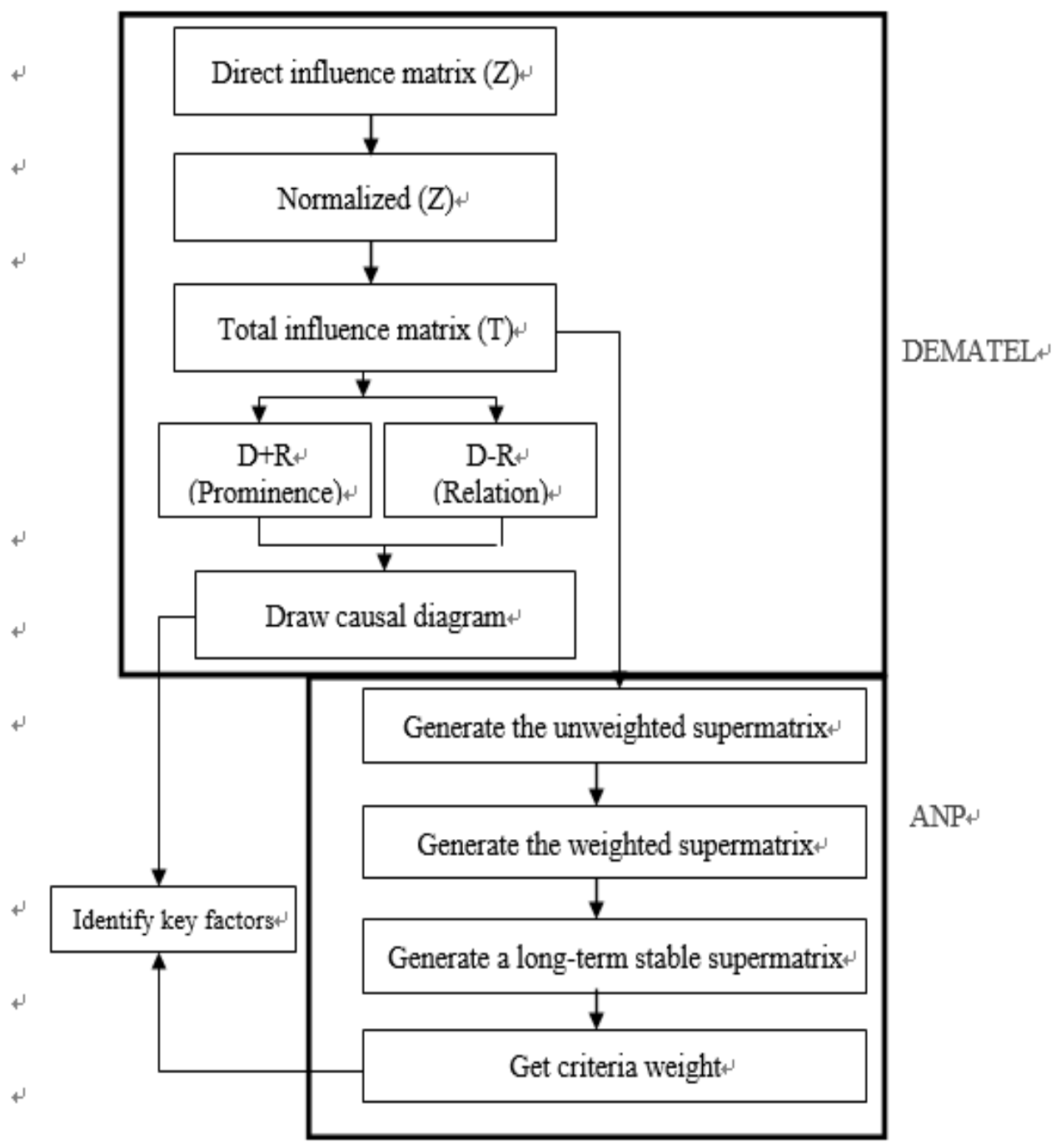

Figure 1: DEMATEL - based ANP 
According to the COVID-19 key decision-making network in Taiwan (https://covid19.mohw.gov.tw/), the factors for successful epidemic prevention are SARS experience, Central Epidemic Command Center, Information Transparency, Good resource allocation, Timely border control, Smart Community transmission prevention, Advanced medical technology, Good citizenship. The preliminary research structure is determined as shown in Figure 1.

This study selected 11 experts with professional knowledge and practical experience from industry, academia and research (Table 1) to participate in filling out the direct influence matrix, and provide complete professional opinions. Through 11 expert influence matrix questionnaires, the average direct influence matrix is obtained (Table 2).

Through the normalization of the direct influence matrix, we generate the total influence matrix (Table 3) using the formula of $\mathrm{T}=\mathrm{X}(\mathrm{I}-\mathrm{X})^{-1}$.

Table 1: Background information of experts

\begin{tabular}{|c|c|c|c|}
\hline Expert & Affiliation & Title & Degree \\
\hline 1 & University & Professor & Ph.D. \\
\hline 2 & Company & Director & Ph.D. \\
\hline 3 & Research unit & Supervisor & Master \\
\hline 4 & Company & Director & Ph.D. \\
\hline 5 & University & Student & Master \\
\hline 6 & Company & Manager & Ph.D. \\
\hline 7 & Medical unit & Dean & Ph.D. \\
\hline 8 & Research unit & Supervisor & Ph.D. \\
\hline 9 & Company & Director & Ph.D. \\
\hline 10 & Company & Manager & Ph.D. \\
\hline 11 & Company & Director & Ph.D. \\
\hline
\end{tabular}


Table 2: Direct influence matrix

\begin{tabular}{|c|c|c|c|c|c|c|c|c|}
\hline $\mathbf{Z}$ & $\mathbf{T 1}$ & $\mathbf{T 2}$ & $\mathbf{T 3}$ & $\mathbf{D 1}$ & $\mathbf{D 2}$ & $\mathbf{D 3}$ & $\mathbf{R 1}$ & $\mathbf{R 2}$ \\
\hline $\mathbf{T 1}$ & 0 & 1.636 & 1.545 & 1.545 & 1.545 & 1.182 & 1.636 & 1.636 \\
\hline $\mathbf{T 2}$ & 1.273 & 0 & 1.182 & 1.091 & 0.818 & 1.091 & 1.273 & 1.091 \\
\hline $\mathbf{T 3}$ & 1.273 & 1.364 & 0 & 1.000 & 1.091 & 1.364 & 1.455 & 1.727 \\
\hline $\mathbf{D 1}$ & 1.091 & 1.000 & 1.182 & 0 & 0.727 & 1.818 & 1.455 & 1.455 \\
\hline $\mathbf{D 2}$ & 1.364 & 1.000 & 1.364 & 0.818 & 0 & 1.364 & 1.545 & 1.000 \\
\hline $\mathbf{D 3}$ & 1.636 & 1.273 & 1.455 & 1.182 & 1.455 & 0 & 1.545 & 1.182 \\
\hline $\mathbf{R 1}$ & 1.455 & 1.000 & 1.182 & 1.273 & 1.727 & 1.545 & 0 & 1.273 \\
\hline $\mathbf{R 2}$ & 1.091 & 0.909 & 1.545 & 1.000 & 1.000 & 1.091 & 1.182 & 0 \\
\hline
\end{tabular}

Table 3: Total influence matrix $(T)$

\begin{tabular}{|c|c|c|c|c|c|c|c|c|c|}
\hline $\mathbf{T}=\mathbf{X}(\mathbf{I}-\mathbf{X})^{-1}$ & $\mathbf{T 1}$ & $\mathbf{T} 2$ & $\mathbf{T 3}$ & $\mathbf{D 1}$ & $\mathbf{D 2}$ & $\mathbf{D 3}$ & $\mathbf{R 1}$ & $\mathbf{R 2}$ & $\mathbf{D}$ (Row sum) \\
\hline $\mathbf{T 1}$ & 0.672 & 0.738 & 0.816 & 0.714 & 0.750 & 0.787 & 0.862 & 0.817 & 6.156 \\
\hline $\mathbf{T 2}$ & 0.618 & 0.462 & 0.624 & 0.541 & 0.548 & 0.615 & 0.661 & 0.614 & 4.681 \\
\hline $\mathbf{T 3}$ & 0.698 & 0.646 & 0.608 & 0.603 & 0.643 & 0.716 & 0.760 & 0.742 & 5.416 \\
\hline $\mathbf{D 1}$ & 0.659 & 0.594 & 0.679 & 0.495 & 0.591 & 0.724 & 0.732 & 0.695 & 5.169 \\
\hline $\mathbf{D 2}$ & 0.667 & 0.584 & 0.679 & 0.555 & 0.516 & 0.676 & 0.726 & 0.648 & 5.051 \\
\hline $\mathbf{D 3}$ & 0.757 & 0.669 & 0.759 & 0.645 & 0.701 & 0.637 & 0.803 & 0.734 & 5.705 \\
\hline $\mathbf{R 1}$ & 0.727 & 0.632 & 0.722 & 0.637 & 0.705 & 0.746 & 0.659 & 0.723 & 5.552 \\
\hline R2 & 0.604 & 0.539 & 0.651 & 0.532 & 0.561 & 0.614 & 0.653 & 0.521 & 4.676 \\
\hline R (Column sum $)$ & 5.402 & 4.864 & 5.538 & 4.723 & 5.015 & 5.515 & 5.856 & 5.494 & \\
\hline
\end{tabular}


In the total influence matrix, the sum of the Row is D, the sum of the Column is R. $\mathrm{D}+\mathrm{R}$ is the Prominence; the higher the value, the higher the importance. $\mathrm{D}-\mathrm{R}$ is the Relation; a positive value indicates an active influencer. The larger the value of D$\mathrm{R}$, the higher the degree of direct influence on other factors. On the contrary, the negative value belongs to the affected person, and the smaller the value, the higher the degree of influence by other factors. The importance and relevance of each factor according to the influence matrix are calculated as shown in Table 4 . We then draw a causal diagram as shown in Figure 2 according to Table 5.

Figure 2 shows that the key factors are SARS experience, Open and transparent information, Smart community transmission prevention, and Central Epidemic Command Center. In order to prove the correctness of the above figure, the total impact matrix of DEMATEL is used as the production criterion. Using the matrix in Table 1 as the basis to self-multiply until convergence produces the ultimate supermatrix as shown in Table 6 . The relative weights are obtained; for example, the weights of $\mathrm{T} 1$ and $\mathrm{R} 2$ are 0.145 and 0.111 respectively.

Table 4: Values of prominence and relation

\begin{tabular}{|c|c|c|c|c|}
\hline Criteria & D (Row sum) & R (Column sum) & D+R (Prominence) & D-R (Relation) \\
\hline T1 & 6.156 & 5.402 & 11.558 & 0.754 \\
\hline T2 & 4.681 & 4.864 & 9.546 & -0.183 \\
\hline T3 & 5.416 & 5.538 & 10.954 & -0.121 \\
\hline D1 & 5.169 & 4.723 & 9.892 & 0.446 \\
\hline D2 & 5.051 & 5.015 & 10.066 & 0.036 \\
\hline D3 & 5.705 & 5.515 & 11.221 & 0.190 \\
\hline R1 & 5.552 & 5.856 & 11.408 & -0.303 \\
\hline R2 & 4.676 & 5.494 & 10.170 & -0.817 \\
\hline
\end{tabular}


Table 5: The supermatrix

\begin{tabular}{|c|c|c|c|c|c|c|c|c|}
\hline $\mathbf{W 4}$ & $\mathbf{T 1}$ & $\mathbf{T 2}$ & $\mathbf{T 3}$ & $\mathbf{D 1}$ & $\mathbf{D 2}$ & $\mathbf{D 3}$ & $\mathbf{R 1}$ & $\mathbf{R 2}$ \\
\hline $\mathbf{T 1}$ & 0.145 & 0.145 & 0.145 & 0.145 & 0.145 & 0.145 & 0.145 & 0.145 \\
\hline $\mathbf{T 2}$ & 0.111 & 0.111 & 0.111 & 0.111 & 0.111 & 0.111 & 0.111 & 0.111 \\
\hline $\mathbf{T 3}$ & 0.128 & 0.128 & 0.128 & 0.128 & 0.128 & 0.128 & 0.128 & 0.128 \\
\hline $\mathbf{D 1}$ & 0.122 & 0.122 & 0.122 & 0.122 & 0.122 & 0.122 & 0.122 & 0.122 \\
\hline $\mathbf{D 2}$ & 0.119 & 0.119 & 0.119 & 0.119 & 0.119 & 0.119 & 0.119 & 0.119 \\
\hline $\mathbf{D 3}$ & 0.135 & 0.135 & 0.135 & 0.135 & 0.135 & 0.135 & 0.135 & 0.135 \\
\hline $\mathbf{R 1}$ & 0.131 & 0.131 & 0.131 & 0.131 & 0.131 & 0.131 & 0.131 & 0.131 \\
\hline $\mathbf{R 2}$ & 0.111 & 0.111 & 0.111 & 0.111 & 0.111 & 0.111 & 0.111 & 0.111 \\
\hline
\end{tabular}

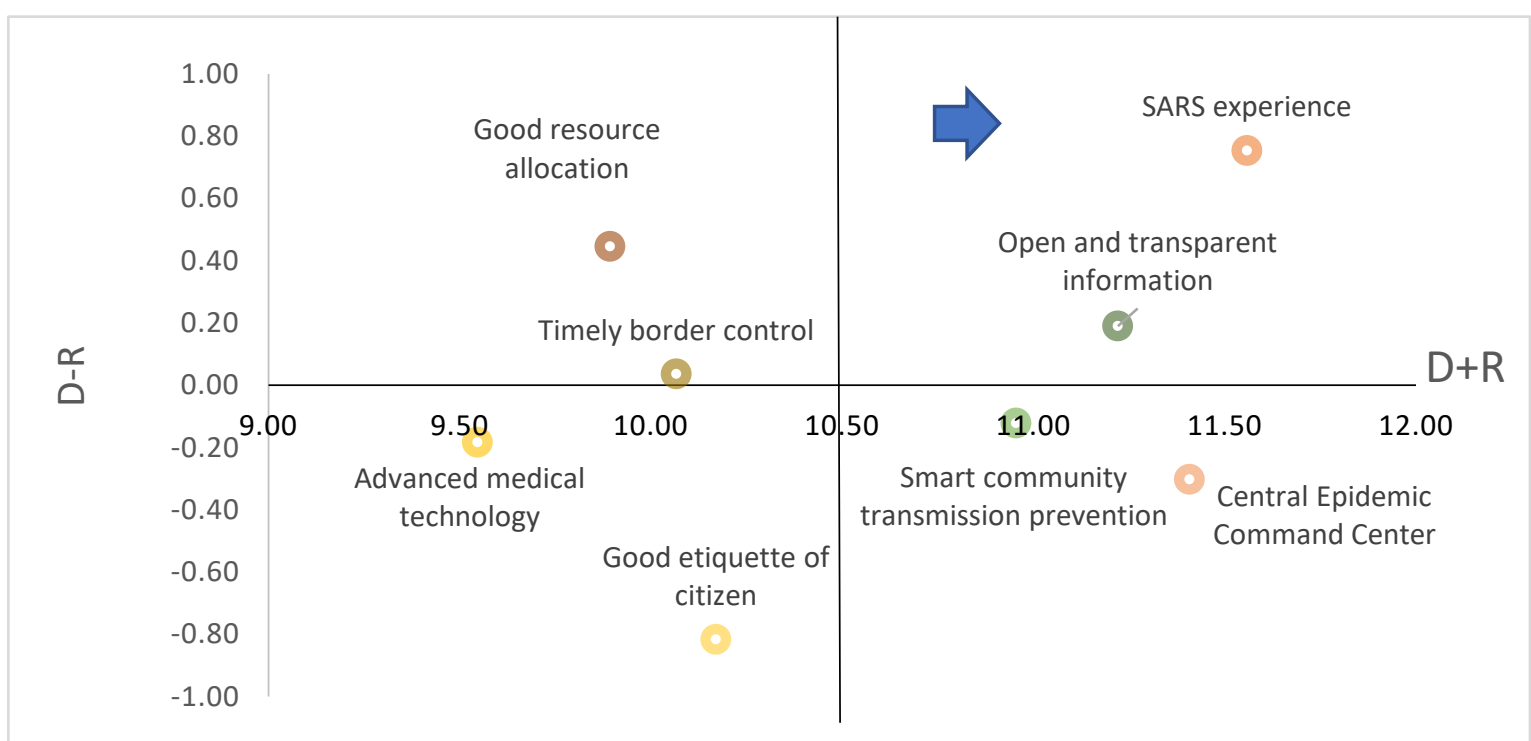

Figure 2: Causal diagram 
The sum of the weight levels of DEMATEL and ANP are shown in Table 6 . According to the final ranking in Table 6, the key factors in sequence that affect the fight against COVID-19 in Taiwan are determined by this study as SARS experience (T1), Open and transparent information (D3), Central Epidemic Command Center (R1), Smart community transmission prevention (T3).

Table 6: Sorting the factors

\begin{tabular}{|c|c|c|c|c|c|}
\hline Criteria & Factors & DEMATEL & D-ANP & Score & $\begin{array}{c}\text { Final } \\
\text { sort }\end{array}$ \\
\hline T1 & SARS experience & 1 & 1 & 2 & 1 \\
\hline T2 & Advanced medical technology & 8 & 8 & 16 & 8 \\
\hline T3 & Smart community transmission prevention & 4 & 4 & 8 & 4 \\
\hline D1 & Good resource allocation & 7 & 5 & 12 & 5 \\
\hline D2 & Timely border control & 6 & 6 & 12 & 5 \\
\hline D3 & Open and transparent information & 3 & 2 & 5 & 2 \\
\hline R1 & Central Epidemic Command Center & 2 & 3 & 5 & 2 \\
\hline R2 & Good citizenship & 5 & 7 & 12 & 5 \\
\hline
\end{tabular}

\section{Conclusion}

In this study, all the factors for successful epidemic prevention listed by the Ministry of Health and Welfare of Taiwan, namely, "SARS experience" (Figure 3), "Central Epidemic Command Center", "Open and Transparent Information", "Good Resource Allocation", "Timely Border Control", "Smart Community Epidemic Prevention", "Advanced Medical Technology", "Good Citizenship" are considered. It was emphasized that the success of epidemic prevention depends on the concerted efforts of the central and local governments to fight the pandemic and the highquality cooperation of the people. 


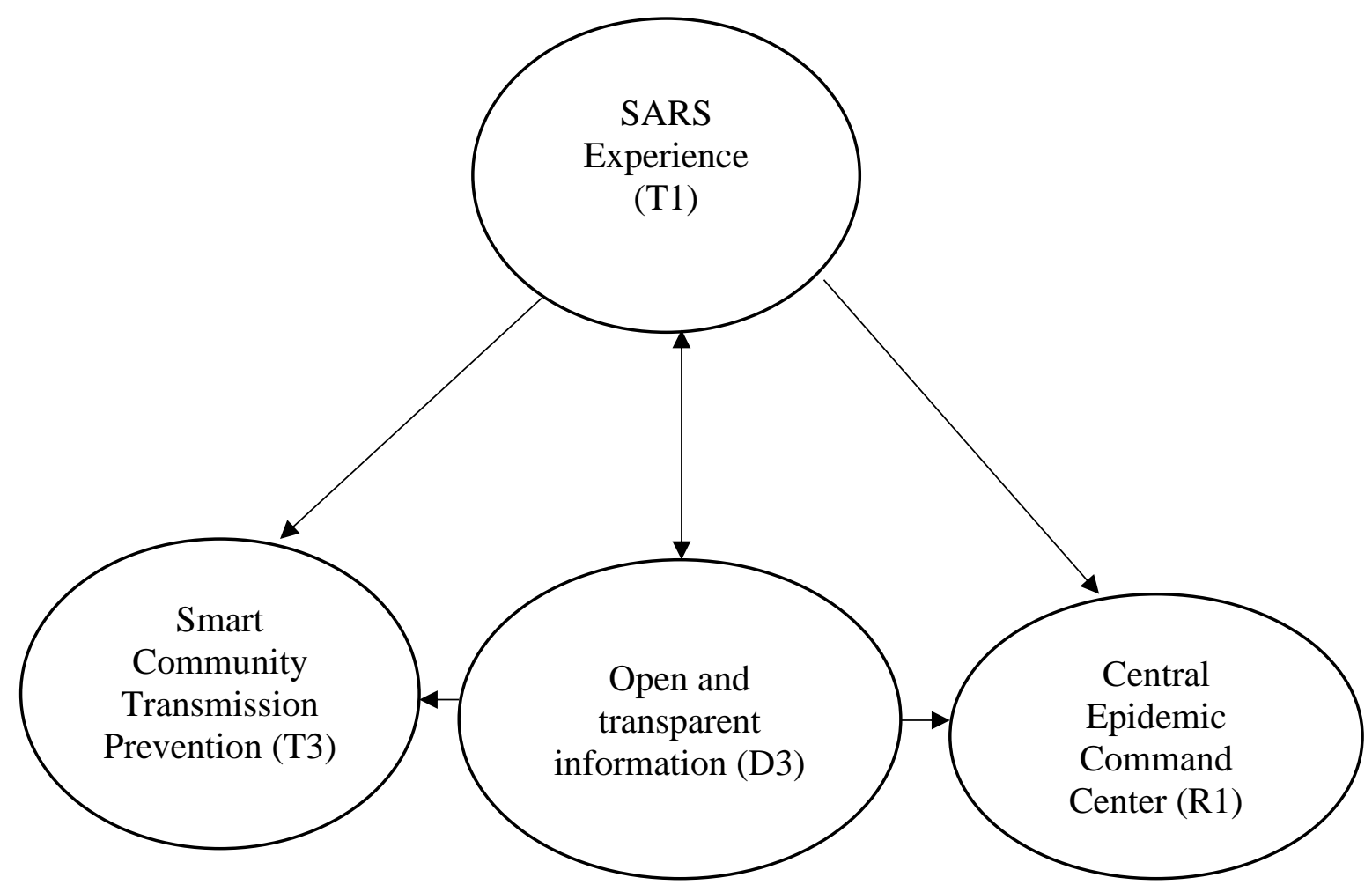

Figure 3: SARS experience

Furthermore, the key factors for successful epidemic prevention are prioritized. Specifically, Open and transparent information (D3), Central Epidemic Command Center (R1) and Smart community transmission prevention (T3) are affected by SARS experience (T1), as shown in Figure 5. In addition, the study confirms that the high quality of the citizens is the most vulnerable factor, because the SARS experience and the transparency of information have enabled the citizens to handle their own affairs and protect themselves and their families under the leadership of the Central Epidemic Center.

The study demonstrates that the success of COVID-19 prevention actually comes from advanced medical technology, good resource allocation, timely border control, experience from SARS, and the decisions made by the Central Epidemic Center. 


\section{References}

[1] Cheng, H. Y., Li, S. Y. and Yang, C. H. (2020). Initial rapid and proactive response for the COVID-19 outbreak: Taiwan's experience. Journal of the Formosan Medical Association, 119(4), pp. 771 - 773.

[2] Chin, A. W. H., Chu, J. T. S., Perera, M. R. A., Hui, K. P. Y., Yen, H.-L., Chan, M. C. W., Peiris, M. and Poon, L. L. M. (2020). Stability of SARS-CoV2 in different environmental conditions. The Lancet Microbe, 1(1), p. e10.

[3] Hsieh C. L., Goldsmith, J. A., Schaub, J. M. et al. (2020). Structure-based design of prefusion-stabilized SARS-CoV-2 spikes. Science, 18(6510), pp. $1501-1505$.

[4] Huang, E. J. (2020). Herbalife is making a difference in nutrition for Taiwan. Taiwan Business Topics, 50(6), p. 2.

[5] Husnayain, A., Fuad, A. and Su, E. C. Y. (2020). Applications of google search trends for risk communication in infectious disease management: A case study of COVID-19 outbreak in Taiwan. Interational Journal of Infectious Diseases, 95(3), pp. $221-223$.

[6] Ko, J. Y., Danielson, M. L., Town, M., Derado, G., Greenland, K. J., Kirley, P. D., Alden, N. B., Yousey-Hindes, K., Anderson, E. J., Ryan, P. A., Kim, S., Lynfield, R., Torres, S. M., Barney, G. R., Bennett, N. M., Sutton, M., H. Talbot, K., Hill, M., Hall, $\quad$ A. J., Fry, A. M., Garg, S. and Kim, L. for the COVID-NET Surveillance Team (2020). Risk factors for COVID-19associated hospitalization: COVID-19-associated hospitalization surveillance network and behavioral risk factor surveillance system. Clinical Infectious Diseases, in press.

[7] Lee, W. C. (2020).Taiwan's experience in pandemic control: Drawing the right lessons from SARS outbreak. Journal of the Chinese Medical Association, 83(7), pp. $622-623$.

[8] Lin, C., Braund, W. E., Auerbach, J., Chou, J. H., Teng, J. H., Tu, P. and Mullen, J. (2020). Policy decisions and use of information technology to fight coronavirus disease, Taiwan. Emerging Infectious Diseases, 26(7), pp. 1506 1512.

[9] Peng, A. P. (2020). Taiwan's response to COVID-19 pandemic. Master's Projects/Capstones, Arts in Asia Pacific Studies, University of San Francisco.

[10] Summers, J., Cheng, H.-Y., Lin, H.-H. et al. (2020). Potential lessons from the Taiwan and New Zealand health responses to the COVID-19 pandemic. The Lancet Regional Health - Western Pacific, 4(November), pp. 1 - 6. 